Pure-injectivity and model theory for G-sets

Rajani, Ravi and Prest, Mike

2008

MIMS EPrint: 2008.82

Manchester Institute for Mathematical Sciences

School of Mathematics

The University of Manchester

\footnotetext{
Reports available from: http://eprints.maths.manchester.ac.uk/

And by contacting: The MIMS Secretary

School of Mathematics

The University of Manchester

Manchester, M13 9PL, UK
}

ISSN 1749-9097 


\title{
Pure-injectivity and model theory for $G$-sets
}

\author{
Ravi Rajani and Mike Prest, \\ School of Mathematics, \\ Alan Turing Building, \\ University of Manchester, \\ Manchester, M13 9PL, \\ UK
}

September 8, 2008

\begin{abstract}
In the model theory of modules the Ziegler spectrum, the space of indecomposable pure-injective modules, has played a key role. We investigate the possibility of defining a similar space in the context of $G$-sets where $G$ is a group.
\end{abstract}

\section{Overview}

In this paper we investigate the possibility of defining a Ziegler Spectrum for $G$-sets where $G$ is a group. The idea of a Ziegler spectrum comes from the model theory of modules (see [13], [9] or, for a recent account, [8]). Given a ring $R$, the Ziegler spectrum for $R$ is a topological space whose points are the isomorphism types of indecomposable pure-injective $R$-modules and whose closed sets correspond to theories in the language of $R$-modules axiomatised by universal implications of positive primitive (pp) formulas. This space has proved to be very useful in the model theory of modules and has applications to the representation theory of rings and algebras.

In the context of modules, there is an key equivalence between the category of positive-primitively-defined imaginaries and the category of finitely 
presented functors on finitely presented modules. In the paper [10] such an equivalence is established for general locally finitely presented categories, though in this, non-additive, context it is the positive existential formulas which take the role played by positive primitive formulas for modules. Positive existential formulas are formulas built up from atomic formulas using the logical operations of conjunction, disjunction, and existential quantification. A coherent theory (a "basic theory" in the terminology of [1]) is a theory axiomatised by formulas of the form

$$
\forall \boldsymbol{x}(\varphi(\boldsymbol{x}) \rightarrow \psi(\boldsymbol{x}))
$$

where $\varphi$ and $\psi$ are positive existential. A definable subcategory of a locally finitely presented category (which will be an elementary class in a first-order language, see, e.g. [1]) is the subcategory of models of a coherent theory (in that language). (This terminology is slightly different from that in the additive context where "definable" means axiomatised by implications between pp formulas; the difference in the additive case between this more restricted notion and that of coherent theory is, however, of little consequence and often disappears.) The main objective of this paper is to find a set of $G$-sets and a topology on this set whose closed subsets correspond to coherent theories of $G$-sets. In other words, we want to define a Ziegler spectrum for $G$-sets.

The category $G$-Set of (left) $G$-sets for a group $G$ is a well behaved locally finitely presented category. It can be thought of as the category of covariant set-valued functors on the category $\{G\}$ which has one object such that $\operatorname{Hom}(G, G)=G$ and composition of maps is simply group multiplication. It is a locally finitely presented topos (by $[1,1.12]$ ) where the regular left $G$-set (which is the functor $\operatorname{Hom}(G,-):\{G\} \rightarrow$ Set represented by the unique object $G$ ) is a strong finitely presented generator. A $G$-set is said to be indecomposable if it is not the coproduct of two proper sub- $G$-sets. Coproducts in $G$-Set are simply disjoint unions. Clearly a $G$-set is indecomposable if and only if it is transitive, and every $G$-set decomposes into a coproduct of indecomposable $G$-sets.

The notions of purity and pure-injectivity (atomic compactness) for $G$ sets were studied by Banaschewski [2] and the initial indication was that there were clear parallels between $G$-sets and modules (e.g. the existence of pureinjective hulls [2, Prop. 3]). In the first part of this paper we extend some of these parallels as well as highlight some important differences. For example, we prove a quantifier elimination result (Theorem 2.5) which says that any 
formula in the language of $G$-sets is equivalent to a boolean combination of quantifier-free formulas and certain sentences which look rather like the "invariant conditions" for modules. This result is probably known but, as far as we are aware, has not been published previously. We use this theorem to show that, as in the modules case, every $G$-set embeds elementarily in its pure-injective hull (Lemma 3.6).

In the case of modules, one shows that definable subcategories are determined by the indecomposable pure-injective modules they contain. In particular, a non-trivial definable subcategory of modules must contain a (non-trivial) indecomposable pure-injective module. One can show this by starting with any non-trivial module $M$ in the definable subcategory $\mathbf{D}$. The pure-injective hull of $M$, denoted $H(M)$ will also be in D. If $H(M)$ decomposes into a direct sum of indecomposables, then $H(M)$ will contain an indecomposable pure-injective direct summand which will also be in $\mathbf{D}$. If $H(M)$ does not decompose into indecomposables, it will, nevertheless, be elementarily equivalent to a pure-injective module which does so decompose. That module will also be in $\mathbf{D}$ and it has an indecomposable pure-injective direct summand which is in $\mathbf{D}([13,6.9])$.

This argument does not work in the case of $G$-sets. Despite the fact that every $G$-set decomposes into indecomposables, there is a pure-injective $G$-set which does not have an indecomposable pure-injective component (Corollary 3.17). Indeed, we will show that there are non-trivial definable subcategories which contain no non-trivial indecomposables whatsoever (Proposition 4.2). Although this implies that the set of indecomposable pureinjectives is not the right set of $G$-sets on which to define a Ziegler topology (whose closed sets correspond to coherent theories), there is a larger set which does work. However, an explicit description of the resulting space in specific examples appears to be hard.

The work reported in this paper forms part of the doctoral thesis of the first author, who has been supported by a MATHLOGAPS Marie Curie Fellowship (MEST-CT- 2004-504029). 


\section{Basic facts about the category $G$-Set}

\subsection{Finitely presented and injective $G$-sets}

If $\mathcal{C}$ is a category with directed colimits then an object $C$ of $\mathcal{C}$ is said to be finitely presented if the hom-functor $(C,-)$ commutes with directed colimits. In the case that $\mathcal{C}$ is a variety this reduces to the usual notion of "finitely generated and finitely related" (see, e.g., $[1,3.10]$ ). It is easy to check that, in the category of $G$-sets, the finitely presented objects have the following characterisation.

Proposition 2.1. A G-set $X$ is finitely presented if and only if $X$ has finitely many orbits and the stabiliser of every element is finitely generated.

In the additive case the injective objects play an important role. First, they are obvious examples of pure-injective objects. Second, each module category has a full embedding into a certain abelian functor category under which the pure-injective modules exactly correspond to the injective functors (see Section 3.1). Using that embedding is the shortest route to proving many of the basic properties of pure-injective modules. We will see that such an embedding is not available in the context of $G$-sets. In any case we note the description of the injective $G$-sets (and, dually, the projective $G$-sets) as well as that of injective hull.

Proposition 2.2.

(a) $A G$-set $X$ is injective if and only if there is some element $x \in X$ with $\operatorname{Stab}(x)=G$.

(b) $X$ is projective if and only if for every element $x \in X, \operatorname{Stab}(x)=1$.

Proof. (a) Suppose that $X$ is injective. Let $\{*\}$ be the one-point $G$-set with $\operatorname{Stab}(*)=G$. $X$ embeds into $X \amalg\{*\}$. By injectivity of $X$ there is a map $f: X \amalg\{*\} \rightarrow X$ of $G$-sets such that $f \uparrow_{X}=\operatorname{id}_{X}$. Let $x=f(*)$. Then for any $g \in G, g x=f(g *)=f(*)=x$. So $\operatorname{Stab}(x)=G$.

Conversely, suppose that $x_{0} \in X$ has $G$ as its stabiliser. Let $X \subseteq Y$ be an inclusion of $G$-sets. Then we can define a map $f: Y \rightarrow X$ by $f(x)=x$ for all $x \in X$ and $f(y)=x_{0}$ for $y \in Y \backslash X$. Clearly $f$ is a map of $G$-sets which splits the embedding $X \subseteq Y$.

(b) Suppose that $X$ is projective. Let $Y=\bigsqcup_{x \in X} G_{x}$ be the disjoint union of copies of the regular left $G$-set $G$ indexed by $X$. Then there is an epimorphism $f: Y \rightarrow X$ which takes the identity in $G_{x}$ to $x \in X$. Since 
$X$ is projective, there is a map $f^{\prime}: X \rightarrow Y$ such that $f f^{\prime}=\mathrm{id}_{X}$. Suppose $g x=x$ in $X$. Then $g f^{\prime}(x)=f^{\prime}(g x)=f^{\prime}(x)$ in $G_{x}$ which implies that $g=1$.

Conversely, suppose that the stabiliser of every element in $X$ is trivial. Then $X$ must decompose as $\bigsqcup_{i} G_{i}$ where $G_{i}=G$ for every $i$. Suppose we have an epimorphism $f: Y \rightarrow X$. Let $1_{i}$ be the identity of $G_{i}$ and let $f^{\prime}\left(1_{i}\right)$ be an element of $f^{-1}\left\{1_{i}\right\}$. Then $f^{\prime}: X \rightarrow Y$ which takes $g \in G_{i}$ to $g f^{\prime}\left(1_{i}\right) \in Y$ is a section of $f$. So $X$ must be projective.

\section{Corollary 2.3.}

(a) $\{*\}$ is the unique indecomposable injective $G$-set and every other injective $G$-set has the form $X \amalg\{*\}$.

(b) The regular left $G$-set $G$ is the unique indecomposable projective object (which is also a projective generator for $G$-Set) and any other projective $G$-set is isomorphic to a disjoint union of copies of $G$.

Given a $G$-set $X$ which is not injective, one can always embed $X$ into the injective object $X \amalg\{*\}$. This is in fact the injective hull of $X$. The injective hull of an object $X$ is an injective object which contains $X$ as an essential subobject. An inclusion $X \subseteq Y$ is essential if any map $f: Y \rightarrow Z$ with $f \uparrow_{X}$ an embedding is itself an embedding. It is easy to deduce that the injective hull of $X$ is unique up to isomorphism over $X$ (thereby justifying the use of the definite article).

Proposition 2.4. Let $X$ be a noninjective G-set. Then, $X \amalg\{*\}$ is the injective hull of $X$.

Proof. Let $f: X \amalg\{*\} \rightarrow Y$ be a map such that $f \uparrow_{X}$ is an injection. Suppose $f$ is not an injection. Then there is an $x_{0} \in X$ with $f(*)=f\left(x_{0}\right)$. Since $X$ is not injective, there is a $g \in G$ such that $g x_{0} \neq x_{0}$. Now $f\left(x_{0}\right)=f(*)=$ $f(g *)=g f(*)=g f\left(x_{0}\right)=f\left(g x_{0}\right)$. But since $f \uparrow_{X}$ is an injection, we must have $x_{0}=g x_{0}$ which is a contradiction. So the embedding $X \subseteq X \amalg\{*\}$ is essential.

\subsection{Formulas in the language of $G$-sets}

As in the case of modules over a ring, there is a natural language for $G$-sets in which we can prove a quantifier elimination result. This language has function symbols corresponding to the elements of $G$ as its only nonlogical symbols. We denote this language by $\mathcal{L}_{G}$. We use the same notation for 
these function symbols as for elements of $G$ and no confusion should arise. The theory of $G$-sets, $T_{G}$, is axiomatised by the sentences

$$
\begin{gathered}
\forall x(1 x=x) \\
\forall x((g h) x=g(h(x)))
\end{gathered}
$$

In any $G$-set we can define the following sets.

$$
\begin{gathered}
\operatorname{supp}(g)=\{x: g x \neq x\} \\
\operatorname{fix}(g)=\{x: g x=x\}
\end{gathered}
$$

For a tuple $\boldsymbol{g}$ from $G$, we define

$$
\begin{aligned}
\operatorname{supp}(\boldsymbol{g}) & =\bigcap_{i} \operatorname{supp}\left(g_{i}\right) \\
\operatorname{fix}(\boldsymbol{g}) & =\bigcap_{i} \operatorname{fix}\left(g_{i}\right)
\end{aligned}
$$

Theorem 2.5. Every formula of $\mathcal{L}_{G}$ is equivalent modulo $T_{G}$ to a Boolean combination of formulas of the form $"|\operatorname{supp}(\boldsymbol{g}) \cap \operatorname{fix}(\boldsymbol{h})| \geq k ", " x \in \operatorname{supp}(\boldsymbol{g}) \cap$ fix $(\boldsymbol{h}) "$, and $g x=y$.

Proof. We can eliminate one existential quantifier at a time. First consider the formula

$$
\exists x\left(x \in \operatorname{supp}(\boldsymbol{g}) \cap \operatorname{fix}(\boldsymbol{h}) \wedge s_{1} y=x \wedge s_{2} y=x \wedge s_{1}^{\prime} y^{\prime}=x \wedge s_{2}^{\prime} y^{\prime}=x\right)
$$

with free variables $y, y^{\prime}$. With a little thought it can be seen that this formula is equivalent to

$$
y \in \operatorname{supp}\left(s_{1}^{-1} \boldsymbol{g} s_{1}\right) \cap \operatorname{fix}\left(s_{1}^{-1} \boldsymbol{h} s_{1}, s_{2}^{-1} s_{1}, s_{1}^{-1} s_{2}^{\prime}\left(s_{1}^{\prime}\right)^{-1} s_{2}\right) \wedge y=s_{1}^{-1} s_{1}^{\prime} y^{\prime}
$$

With this example in mind, it should be clear that it is sufficient to show that a formula $\psi\left(y_{1}, \ldots, y_{n}\right)$ of the form

$$
\exists x\left(x \in \operatorname{supp}(\boldsymbol{g}) \cap \operatorname{fix}(\boldsymbol{h}) \wedge \bigwedge_{i=1}^{n}\left(y_{i} \neq s_{i 1} x \wedge \cdots \wedge y_{i} \neq s_{i \nu_{i}} x\right)\right)
$$

is of the required form. Suppose that this formula is true in a $G$-set $X$. Suppose that for each $l \in\{1, \ldots, n\}, J_{l}$ is a set of indices from $\left\{1, \ldots, \nu_{l}\right\}$ such that the elements $s_{l j}^{-1} y_{l}$ for $j \in J_{l}, l=1, \ldots, n$ are all distinct and 
such that each $s_{l j}^{-1} y_{l}$ is in $\operatorname{supp}(\boldsymbol{g}) \cap \operatorname{fix}(\boldsymbol{h})$. Moreover, take the collection of sets $J_{l}$ to be a maximal such collection in the sense that for any $l$, if $j \notin J_{l}$, then either $s_{l j}^{-1} y_{l} \notin \operatorname{supp}(\boldsymbol{g}) \cap \operatorname{fix}(\boldsymbol{h})$ or $s_{l j}^{-1} y_{l}=s_{i k}^{-1} y_{i}$ for some $i$ and $k \in J_{i}$. Then the formula $\psi$ is true in $X$ when $|\operatorname{supp}(\boldsymbol{g}) \cap \operatorname{fix}(\boldsymbol{h})| \geq \sum_{l=1}^{n}\left|J_{l}\right|+1$. But, since there are only finitely many different variations on this particular situation, we can enumerate all the possibilities so that $\psi$ can be rewritten as the disjunction of all formulas

$$
\begin{gathered}
\bigwedge_{l=1}^{n} \bigwedge_{j \in J_{l}}\left(s_{l j}^{-1} y_{l} \in \operatorname{supp}(\boldsymbol{g}) \cap \operatorname{fix}(\boldsymbol{h})\right) \\
\wedge \bigwedge_{l \neq m} \bigwedge_{j \neq j^{\prime} \in J_{l}} \bigwedge_{k \in J_{m}}\left(s_{l j}^{-1} y_{l} \neq s_{m k}^{-1} y_{m} \wedge s_{l j}^{-1} y_{l} \neq s_{l j^{\prime}}^{-1} y_{l}\right) \\
\bigwedge_{l=1}^{n} \bigwedge_{j \notin J_{l}}\left(s_{l j}^{-1} y_{l} \notin \operatorname{supp}(\boldsymbol{g}) \cap \operatorname{fix}(\boldsymbol{h}) \vee \bigvee_{m=1}^{n} \bigvee_{k \in J_{m}} s_{l j}^{-1} y_{l}=s_{m k}^{-1} y_{m}\right) \\
\wedge|\operatorname{supp}(\boldsymbol{g}) \cap \operatorname{fix}(\boldsymbol{h})| \geq\left(\sum_{l=1}^{n}\left|J_{l}\right|\right)+1
\end{gathered}
$$

where each $J_{l}$ ranges over subsets of $\left\{1, \ldots, \nu_{l}\right\}$. Since ty $\in \operatorname{supp}(\boldsymbol{g}) \cap \operatorname{fix}(\boldsymbol{h})$ is equivalent to $y \in \operatorname{supp}\left(t^{-1} \boldsymbol{g} t\right) \cap \operatorname{fix}\left(t^{-1} \boldsymbol{h} t\right)$ and $t_{1} y_{1}=t_{2} y_{2}$ is equivalent to $y_{1}=t_{1}^{-1} t_{2} y_{2}$, the above formula is of the required form.

This result looks rather like the "pp elimination of quantifiers" result for modules (see, e.g., [9, Cor 2.13]). It is probably known but we were unable to find a reference.

Corollary 2.6. In $\mathcal{L}_{G}$, every sentence is equivalent modulo $T_{G}$ to a Boolean combination of sentences of the form

$$
|\operatorname{supp}(\boldsymbol{g}) \cap \operatorname{fix}(\boldsymbol{h})| \geq k
$$

Corollary 2.7. For any $G$-set $X, \operatorname{Th}(X)$ has quantifier elimination.

\section{Purity and pure-injectivity in $G$-Set}

\subsection{Pure embeddings}

Recall that an inclusion of $G$-sets $X \subseteq Y$ is pure if for every positive primitive formula $\varphi(\boldsymbol{x})$ and tuple $\boldsymbol{a}$ from $X, Y \models \varphi(\boldsymbol{a}) \Rightarrow X \models \varphi(\boldsymbol{a})$. Now a positive 
primitive formula in $\mathcal{L}_{G}$ is equivalent to a formula of the form

$$
x_{1} \in \operatorname{fix}\left(\boldsymbol{g}_{1}\right) \wedge \cdots \wedge x_{n} \in \operatorname{fix}\left(\boldsymbol{g}_{n}\right) \wedge \operatorname{fix}\left(\boldsymbol{h}_{1}\right) \neq \emptyset \wedge \cdots \wedge \operatorname{fix}\left(\boldsymbol{h}_{m}\right) \neq \emptyset
$$

So we have the following proposition, which appears as Lemma 1 in [2].

Proposition 3.1. The extension $X \subseteq Y$ is pure if and only if for any finite tuple $\boldsymbol{g}$ from $G$, $\operatorname{fix}_{Y}(\boldsymbol{g}) \neq \emptyset$ implies $\operatorname{fix}_{X}(\boldsymbol{g}) \neq \emptyset$.

Let $R$-Mod be the category of left $R$-modules and mod- $R$ the category of finitely presented right $R$-modules. For any left $R$-module $M$, one has a functor given on objects by $T_{M}:=-\otimes M \in(\bmod -R, \mathbf{A b})$, and with the obvious action on morphisms. $T$ is a fully faithful functor

\section{$R-\operatorname{Mod} \longrightarrow(\bmod -R, \mathbf{A b})$}

such that a monomorphism $f: M \rightarrow N$ is pure in $R$-Mod if and only if $-\otimes f$ : $(-\otimes M) \rightarrow(-\otimes N)$ is a monomorphism in $(\bmod -R, \mathbf{A b})($ see $[5,7.12, \mathrm{~B} 16])$. Furthermore, a module $M$ is pure-injective if and only if the corresponding functor $T_{M}$ is injective. This functor, which converts the theory of purity in $R$-Mod to the simpler theory around injectivity in the functor category, has been methodologically very useful for the study of purity and the associated model theory, so it is natural to ask whether there is a similar picture for $G$-sets. We show that there is not.

Let $X$ be a right $G$-set and $Y$ a left $G$-set. Then $X \otimes_{G} Y$ is the set of elements $x \otimes y$ factored by the equivalence relation

$$
x \otimes y \sim x^{\prime} \otimes y^{\prime} \Leftrightarrow \exists g \in G \text { s.t. } x^{\prime}=x g \text { and } y^{\prime}=g^{-1} y
$$

(see $[6$, p. 380]). The operation $\otimes$ behaves like a tensor product in the sense that there is a Hom-tensor adjunction. Given a $G$-set $Y$ and a set $E$, we can get a $G^{\text {op }}$-set (right $G$-set) $\operatorname{Hom}(Y, E)$ where the action of $G$ is defined by $(f g)(y)=f(g y)$. If $\varphi: X \otimes Y \rightarrow E$ is a map of sets, we can get a map $\Gamma_{\varphi}: X \rightarrow \operatorname{Hom}(Y, E)$ of $G^{\text {op }}$-sets defined by

$$
\Gamma_{\varphi}(x)(y)=\varphi(x \otimes y)
$$

Conversely, if $\psi: X \rightarrow \operatorname{Hom}(Y, E)$ is a map of $G^{\text {op }}$-sets then there is a map of sets $\Delta_{\psi}: X \otimes Y \rightarrow E$ defined by

$$
\Delta_{\psi}(x \otimes y)=\psi(x)(y)
$$


These maps give an adjunction

$$
\operatorname{Hom}(X \otimes Y, E) \underset{\Delta}{\stackrel{\Gamma}{\rightleftarrows}} \operatorname{Hom}(X, \operatorname{Hom}(Y, E))
$$

As in the case of modules, for any (left) $G$-set $Y$, we get an object $-\otimes Y \in$ $\left(G^{\mathrm{op}}\right.$-set, Set) where $G^{\mathrm{op}}$-set is the category of finitely presented $G^{\mathrm{op}}$-sets. For any $F \in\left(G^{\text {op }}\right.$-set, Set $), F G$ is a $G$-set with action defined by $g x=$ $F\left(l_{g}\right)(x)$ where $l_{g}$ is the $G^{\text {op }}$-endomorphism of $G$ given by left multiplication by $g$. The map $g \otimes y \mapsto g y$ gives an isomorphism $G \otimes Y \cong Y$ of $G$-sets and the map $f \mapsto f(1)$ gives an isomorphism $\operatorname{Hom}(G, X) \cong X$ of $G^{\text {op }}$-sets. Moreover, the map which evaluates a natural transformation at $G$, gives a natural isomorphism

$$
\operatorname{Hom}(-\otimes Y, F) \cong \operatorname{Hom}(Y, F G)
$$

where the right-hand Hom is of (left) $G$-sets.

With the isomorphism $Y \cong G \otimes Y$ it is easy to see that the functor $Y \mapsto-\otimes Y$ is fully faithful so that $G$-Set embeds into $\left(G^{\text {op }}\right.$-set, Set $)$. The situation looks remarkably similar to the modules case. So can we characterise pure embeddings of $G$-sets in terms of the tensor product? Unfortunately the answer to this question is negative.

Proposition 3.2. For any $X \in G^{o p}$-set, the functor $X \otimes-$ preserves monomorphisms.

Proof. Let $f: Y \rightarrow Y^{\prime}$ be a monomorphism of $G$-sets. $(X \otimes f)(x \otimes y)=$ $x \otimes f(y)$. Suppose that $x \otimes f(y)=x^{\prime} \otimes f\left(y^{\prime}\right)$. Then there is a $g \in G$ such that $x^{\prime}=x g$ and $f\left(y^{\prime}\right)=g^{-1} f(y)$. But $f\left(g^{-1} y\right)=g^{-1} f(y)=f\left(y^{\prime}\right)$ implies that $g^{-1} y=y^{\prime}$ since $f$ is assumed to be a monomorphism. Hence $x \otimes y=x^{\prime} \otimes y^{\prime}$.

So the functor $Y \mapsto-\otimes Y$ takes all embeddings of $G$-sets, not just the pure ones, to embeddings of functors.

We say that a $G$-set $X$ is pure-injective if every pure embedding $f: X \rightarrow$ $Y$ is split, i.e. there is a map $g: Y \rightarrow X$ such that $g f=\mathrm{id}_{X}$.

Corollary 3.3. Let $X$ be a pure-injective, noninjective $G$-set. Then $-\otimes X$ is not an injective functor. 
Proof. Since $X$ is noninjective, there is a $G$-set $Y$ and a monomorphism $f$ such that $f: X \rightarrow Y$ is not split. We claim that $-\otimes f$ is a non-split embedding. By above it is certainly an embedding. Suppose it were split. So there is a $\theta:-\otimes Y \rightarrow-\otimes X$ such that $\theta \circ(-\otimes f)=$ id. Now $\theta=-\otimes \theta_{G}$ where $\theta_{G}: Y \rightarrow X$. We have that $\theta_{G} f=\operatorname{id}_{X}$ which is a contradiction.

\subsection{Pure-injective $G$-sets and pure-injective hulls}

A standard result of model theory gives us that a $G$-set $X$ is pure-injective if and only if every atomic type $\Phi(\boldsymbol{x})$ (where $\boldsymbol{x}$ may be infinite) with parameters from $X$ is realised in $X$ (note this is equivalent to every pp-type in finitely many variables being realised, see e.g. Hodges [4, Thm 10.7.1]). It is clear that we need only consider parameter-free types since if $a \in X$ and $g x=a$ is an equation, then $x=g^{-1} a$ is uniquely determined in $X$. So let $\Phi\left(x_{i}\right)_{i}$ be a parameter-free atomic type of $X$. Assume that $\Phi$ is closed under deductive consequences of equations. Define the equivalence relation $\sim$ on the set of variables $\left\{x_{i}\right\}_{i}$ by

$$
x_{j} \sim x_{k} \Leftrightarrow \exists g \in G \text { s.t. } g x_{j}=x_{k} \in \Phi
$$

Suppose that $\sim$ is transitive on $\left\{x_{i}\right\}_{i}$. Then $\Phi$ is equivalent to the type $\Phi^{\prime}\left(x_{0}\right)=\left\{g x_{0}=x_{0}: g x_{0}=x_{0} \in \Phi\right\}$ for any chosen $x_{0}$. For if we have the formula $g x_{j}=x_{k} \in \Phi$, then since $\sim$ is transitive, there are $h, h^{\prime} \in G$ such that $x_{j}=h^{\prime} x_{0}$ and $x_{k}=h x_{0}$ and so since $\Phi$ is assumed to contain all its equational consequences, we have that $h^{-1} g h^{\prime} x_{0}=x_{0} \in \Phi$. Hence this equation is in $\Phi^{\prime}$ and implies $g x_{j}=x_{k}$. So $\Phi$ is equivalent to a type in one variable which corresponds to a subset $S \subseteq G$ with the property that for any finite tuple $\boldsymbol{s}$ from $S$, $\operatorname{fix}_{X}(\boldsymbol{s}) \neq \emptyset$. If $\sim$ is not transitive on $\left\{x_{i}\right\}_{i}$, then $\Phi$ is equivalent to a family of types in one variable (one for each equivalence class). So we have the following characterisation of pure-injectivity for $G$-sets which appears as Proposition 1 in [2].

Proposition 3.4. The $G$-set $X$ is pure-injective (or equationally compact) if whenever $S \subseteq G$ is such that for any finite tuple $\boldsymbol{s}$ from $S$, $\operatorname{fix}_{X}(\boldsymbol{s}) \neq \emptyset$, then $\operatorname{fix}_{X}(S):=\bigcap_{s \in S} \operatorname{fix}_{X}(s) \neq \emptyset$.

Equivalently, a $G$-set $X$ is pure-injective if and only if it satisfies the following condition. 
(3.2.1) If $H \leq G$ is a subgroup such that $\operatorname{fix}_{X}\left(H^{\prime}\right) \neq \emptyset$ for every finitely generated subgroup $H^{\prime} \leq H$, then $\operatorname{fix}_{X}(H) \neq \emptyset$.

We say that a subgroup $S$ can be conjugated into a subgroup $H$ if one of the conjugates of $S$ is contained in $H$. Since a subgroup of $G$ fixes a point of the transitive $G$-set $G / H$ if and only if it can be conjugated into $H$ (because $\left.\operatorname{Stab}(g H)=g H^{-1}\right)$ we have the following

Corollary 3.5. The transitive $G$-set $G / H$ is pure-injective if and only if any subgroup $S \leq G$ whose finitely generated subgroups can be conjugated into $H$, can itself be conjugated into $H$.

We shall say that a subgroup $H \leq G$ is PIP (the pure-injectivity property) if $G / H$ is a pure-injective $G$-set. Clearly 1 and $G$ are both PIP. Also, any finite or normal subgroup of $G$ is PIP.

An extension $X \subseteq Y$ is pure essential if it is pure and whenever $f: Y \rightarrow Z$ is a map such that $f \Upsilon_{X}$ is a pure embedding, then $f$ is an embedding. By our characterisation of pure embeddings for $G$-sets, the $f$ of this definition will in fact be a pure embedding. A $G$-set $Y$ is the pure-injective hull of $X$ if $Y$ is pure-injective and contains $X$ as a pure-essential subobject. The pure-injective hull of $X$ is unique up to isomorphism over $X$ (see [12]). Banaschewski [2] shows that every $G$-set $X$ has a pure-injective hull which we denote by $H(X)$. We will now show that $H(X)$ is elementary equivalent to $X$ (as in the case of modules); also see [11] where it is commented that Mycielski pointed out that, by Theorems 1 and 5 of [7], every $G$-set is an elementary substructure of a pure-injective $G$-set.

Lemma 3.6. The pure-injective hull $H(X)$ of $X$ is elementarily equivalent to $X$ and the embedding of $X$ in $H(X)$ is elementary.

Proof. To see this embed $X$ elementarily in a $\emptyset$-saturated extension $Y$ (so in particular $Y$ is pure-injective and the embedding is pure). The hull $H(X)$ will sit in between $X$ and $Y$ (see Wenzel [12]) and the embedding of $H(X)$ in $Y$ will be pure (since $X \subseteq H(X)$ is pure-essential).

$$
X \underset{\text { pure }}{\longrightarrow} H(X) \underset{\text { pure }}{\longrightarrow} Y
$$

Let $\varphi$ be a sentence of the form $|\operatorname{supp}(\boldsymbol{g}) \cap \operatorname{fix}(\boldsymbol{h})| \geq k$. Clearly $X \models \varphi$ implies $H(X) \models \varphi$. For the converse, if $H(X) \models \varphi$ then so does $Y \models \varphi$. But now since $X \subseteq Y$ is elementary, $X \models \varphi$. By Corollary 2.6 $X \equiv H(X)$. 
Since complete theories of $G$-sets have quantifier elimination, the embedding of $X$ in its pure-injective hull $H(X)$ is elementary (as in the case of modules).

The paper [2] contains a reasonably explicit construction of the pureinjective hull which we now describe. Let $\mathrm{FF}(X)$ be the set of subgroups $H \leq G$ such that for any finitely generated $H^{\prime} \leq H$, $\operatorname{fix}_{X}\left(H^{\prime}\right) \neq \emptyset$. By Zorn's Lemma, any such subgroup $H$ is contained in a maximal one. Let $\mathbb{M}_{X}$ be the set of maximal subgroups $H$ in $\mathrm{FF}(X)$ such that $\operatorname{fix}_{X}(H)=\emptyset$. Note that if $X$ is pure-injective, then $\mathbb{M}_{X}=\emptyset$. Clearly each of the sets $\mathrm{FF}(X)$ and $\mathbb{M}_{X}$ is closed under conjugacy in $G$. Let $\mathbb{I M}_{X}$ be a set of representatives of conjugacy classes of subgroups in $\mathbb{M}_{X}$. Then Banaschewski [2] constructs the pure-injective hull of $X$ as

$$
X \coprod\left(\coprod_{H \in \mathbb{M}_{X}} G / H\right)
$$

In the modules case, if a pure-injective module has an indecomposable direct summand, then it has an indecomposable pure-injective direct summand. So, since every $G$-set decomposes into indecomposables, one might expect that every pure-injective $G$-set contains an indecomposable pure-injective component. This leads one to ask first of all whether some $H \in \mathbb{M}_{X}$ is PIP. We will now show that this is equivalent to a problem about ultrafilters.

For convenience we will write, for any set $A, A^{<\omega}$ for the set of finite tuples from $A$. Fix an infinite $G$-set $X$. Define

$$
\mathbb{L}_{X}=\left\{\operatorname{fix}_{X}(\boldsymbol{g}): \boldsymbol{g} \in G^{<\omega}\right\} \cup\{\emptyset\}
$$

Then $\mathbb{L}_{X}$ is a bounded meet-semilattice (a poset with meets, a top, and a bottom element). If $G=\operatorname{Sym}(X)$ then $\mathbb{L}_{X}$ is the full powerset of $X$. On the other hand if $G$ acts trivially on $X$, so that $G_{x}=G$ for every $x \in X$, then $\mathbb{L}_{X}=\{X, \emptyset\}$.

If $\mathcal{F}$ is a filter of $\mathbb{L}_{X}$, then we define the subgroup

$$
H_{\mathcal{F}}=\{g \in G: \operatorname{fix}(g) \in \mathcal{F}\}
$$

which is an object of $\mathrm{FF}(X)$. The fact that $H_{\mathcal{F}}$ is a subgroup follows from the identity $\operatorname{fix}(g)=\operatorname{fix}\left(g^{-1}\right)$ and the inclusion $\operatorname{fix}(g, h) \subseteq \operatorname{fix}(g h)$ for any $g, h \in G$. That $H_{\mathcal{F}}$ is in $\operatorname{FF}(X)$ follows from the fact that $\emptyset \notin \mathcal{F}$. 
Conversely, if we have a subgroup $H \in \mathrm{FF}(X)$, then we can define the filter

$$
\mathcal{F}(H)=\left\{A \in \mathbb{L}_{X}: A \supseteq \operatorname{fix}(\boldsymbol{h}) \text { for some } \boldsymbol{h} \in H^{<\omega}\right\}
$$

This is the smallest filter containing the set $\{\operatorname{fix}(h): h \in H\}$.

Now,

$$
\begin{aligned}
\mathcal{F}\left(H_{\mathcal{F}}\right) & =\left\{A \in \mathbb{L}_{X}: A \supseteq \operatorname{fix}(\boldsymbol{h}) \text { some } \boldsymbol{h} \in H_{\mathcal{F}}^{<\omega}\right\} \\
& =\left\{A \in \mathbb{L}_{X}: A \supseteq \operatorname{fix}(\boldsymbol{h}) \text { where fix }(\boldsymbol{h}) \in \mathcal{F}\right\}
\end{aligned}
$$

so that, clearly,

$$
\mathcal{F}\left(H_{\mathcal{F}}\right)=\mathcal{F}
$$

Also,

$$
\begin{aligned}
H_{\mathcal{F}(H)} & =\{g \in G: \operatorname{fix}(g) \in \mathcal{F}(H)\} \\
& =\left\{g \in G: \operatorname{fix}(g) \supseteq \operatorname{fix}(\boldsymbol{h}) \text { where } \boldsymbol{h} \in H^{<\omega}\right\}
\end{aligned}
$$

so that

$$
H \subseteq H_{\mathcal{F}(H)}
$$

Lemma 3.7. If $H$ is maximal in $\mathrm{FF}(X)$, then $H=H_{\mathcal{F}(H)}$

We shall say that a filter $\mathcal{F}$ is principal if there is a nonempty subset $Y \subseteq X$ such that

$$
\mathcal{F}=\left\{A \in \mathbb{L}_{X}: A \supseteq Y\right\}
$$

The notation for such a filter is $\langle Y\rangle$. Note that $Y$ need not be in $\mathbb{L}_{X}$ so our terminology deviates slightly from the standard one.

Lemma 3.8. If $\mathcal{F}$ is a principal filter then $\operatorname{fix}\left(H_{\mathcal{F}}\right) \neq \emptyset$.

Proof. Suppose $\mathcal{F}=\langle Y\rangle$. Since fix $\left(H_{\mathcal{F}}\right)=\cap \mathcal{F}$, we have fix $\left(H_{\mathcal{F}}\right) \supseteq Y \neq \emptyset$.

An ultrafilter is a maximal filter. By Zorn's Lemma, any filter is contained in an ultrafilter.

Lemma 3.9. An ultrafilter $\mathcal{U}$ of $\mathbb{L}_{X}$ is principal if and only if $\operatorname{fix}\left(H_{\mathcal{U}}\right) \neq \emptyset$.

Proof. Suppose that $\operatorname{fix}\left(H_{\mathcal{U}}\right) \neq \emptyset$. Then $\mathcal{U}$ is contained in the proper principal filter $\left\langle\operatorname{fix}\left(H_{\mathcal{U}}\right)\right\rangle$ and hence must be equal to it. 
We shall write $G_{(Y)}$ for the point stabiliser of $Y$.

Lemma 3.10. Let $H=G_{(Y)}$ then

(a) $H=H_{\mathcal{F}(H)}$.

(b) $\mathcal{F}(H)=\langle Y\rangle$

Proof. (a) Suppose $h \in H_{\mathcal{F}(H)}$. Then $\operatorname{fix}(h) \in \mathcal{F}(H)$ so that $\operatorname{fix}(h) \supseteq \operatorname{fix}(\boldsymbol{g})$ for some $\boldsymbol{g} \in G_{(Y)}^{<\omega}$. But this means fix $(h) \supseteq Y$ which in turn means that $h \in G_{(Y)}=H$.

(b) Suppose $A \in \mathcal{F}(H)$. Then $A \supseteq \operatorname{fix}(\boldsymbol{h})$ where $\boldsymbol{h} \in G_{(Y)}^{<\omega}$. So $A \supseteq$ $\operatorname{fix}(\boldsymbol{h}) \supseteq Y$. That is $A \in\langle Y\rangle$. Conversely if $A=\operatorname{fix}(\boldsymbol{g}) \supseteq Y$ then $\boldsymbol{g} \in G_{(Y)}^{<\omega}$ so that $A \in \mathcal{F}(H)$.

Lemma 3.11. If $H$ is a maximal subgroup in $\mathrm{FF}(X)$ then $\mathcal{F}(H)=\langle Y\rangle$ if and only if $H=G_{(Y)}$.

Proof. Suppose that $\mathcal{F}(H)=\langle Y\rangle$. Let $h \in H$. Then $\operatorname{fix}(h) \in \mathcal{F}(H)=\langle Y\rangle$ so that $h \in G_{(Y)}$. So $H \subseteq G_{(Y)}$ and by maximality $H=G_{(Y)}$. The converse is Lemma $3.10(\mathrm{~b})$.

Lemma 3.12. Let $\mathcal{U}$ be an ultrafilter. Then $H_{\mathcal{U}}$ is maximal in $\mathrm{FF}(X)$.

Proof. Suppose $H_{\mathcal{U}} \leq H$ where $H \in \mathrm{FF}(X)$. We then have $\mathcal{U}=\mathcal{F}\left(H_{\mathcal{U}}\right) \subseteq$ $\mathcal{F}(H)$ so that $\mathcal{U}=\mathcal{F}(H)$. Now let $h \in H$. Then $\operatorname{fix}(h) \in \mathcal{F}(H)=\mathcal{U}$ so that $h \in H_{\mathcal{U}}$. So we have $H=H_{\mathcal{U}}$.

Lemma 3.13. If $H$ is maximal in $\mathrm{FF}(X)$ then $\mathcal{F}(H)$ is an ultrafilter.

Proof. Let $\mathcal{U}$ be an ultrafilter containing $\mathcal{F}(H)$. Then $H \subseteq H_{\mathcal{F}(H)} \subseteq H_{\mathcal{U}}$. By maximality, $H=H_{\mathcal{U}}$. So $\mathcal{F}(H)=\mathcal{F}\left(H_{\mathcal{U}}\right)=\mathcal{U}$.

The following proposition now follows easily from these lemmas.

Proposition 3.14. There is a bijection between ultrafilters of $\mathbb{L}_{X}$ and maximal subgroups in $\mathrm{FF}(X)$. The nonprincipal ultrafilters correspond to those subgroups in $\mathbb{M}_{X}$.

The group $G$ acts on the space of ultrafilters of $\mathbb{L}_{X}$ by $g \mathcal{U}=\{g A: A \in \mathcal{U}\}$. For any $h_{1}, \ldots, h_{n} \in G, g \operatorname{fix}\left(h_{1}, \ldots, h_{n}\right)=\operatorname{fix}\left(g h_{1} g^{-1}, \ldots, g h_{n} g^{-1}\right)$. This implies that when $H$ is a maximal subgroup in $\mathrm{FF}(X)$,

$$
g \mathcal{F}(H)=\mathcal{F}\left(g H g^{-1}\right)
$$


Proposition 3.15. A maximal subgroup $H \in \mathrm{FF}(X)$ is PIP if and only if, for any filter $\mathcal{F} \subseteq \mathbb{L}_{X}$ such that $\mathcal{F} \subseteq \cup_{g \in G} g \mathcal{F}(H)$, there is a $g_{0} \in G$ such that $\mathcal{F} \subseteq g_{0} \mathcal{F}(H)$.

Proof. $(\Rightarrow)$ Suppose $H$ is a PIP maximal subgroup in $\mathrm{FF}(X)$. Let $\mathcal{F} \subseteq \mathbb{L}_{X}$ be a filter such that $\mathcal{F} \subseteq \cup_{g \in G} g \mathcal{F}(H)$. Then $H_{\mathcal{F}}$ is locally conjugated into $H$. For suppose $s_{1}, \ldots, s_{n} \in H_{\mathcal{F}}$. Then $\operatorname{fix}\left(s_{1}, \ldots, s_{n}\right) \in \mathcal{F}$ and so there is a $g \in G$ such that fix $\left(s_{1}, \ldots, s_{n}\right) \in g \mathcal{F}(H)=\mathcal{F}\left(g H g^{-1}\right)$. So $s_{1}, \ldots, s_{n} \in H_{\mathcal{F}\left(g H g^{-1}\right)}=$ $g H g^{-1}$. Since $H$ is PIP, there is a $g_{0} \in G$ such that $H_{\mathcal{F}} \subseteq g_{0} H g_{0}^{-1}$. So $\mathcal{F}=\mathcal{F}\left(H_{\mathcal{F}}\right) \subseteq \mathcal{F}\left(g_{0} H g_{0}^{-1}\right)=g_{0} \mathcal{F}(H)$.

$(\Leftarrow)$ Suppose $S \leq G$ is locally conjugated into $H$. Then in particular $S \in$ $\mathrm{FF}(X)$. We claim that $\mathcal{F}(S) \subseteq \bigcup_{g \in G} g \mathcal{F}(H)$. For suppose $A \supseteq \operatorname{fix}\left(s_{1}, \ldots, s_{n}\right)$ where $s_{1}, \ldots, s_{n} \in S$. By assumption, there is a $g \in G$ such that $s_{1}, \ldots, s_{n} \in$ $g H g^{-1}$. So fix $\left(s_{1}, \ldots, s_{n}\right) \in \mathcal{F}\left(g H g^{-1}\right)=g \mathcal{F}(H)$ and $A$ must be in $g \mathcal{F}(H)$ which establishes the claim. But this means there is a $g_{0} \in G$ with $\mathcal{F}(S) \subseteq$ $g_{0} \mathcal{F}(H)=\mathcal{F}\left(g_{0} H g_{0}^{-1}\right)$. Hence $S \subseteq H_{\mathcal{F}(S)} \subseteq H_{\mathcal{F}\left(g_{0} H g_{0}^{-1}\right)}=g_{0} H g_{0}^{-1}$.

So the PIP subgroups in $\mathbb{M}_{X}$ correspond to the nonprincipal ultrafilters $\mathcal{U}$ of $\mathbb{L}_{X}$ with the property that

(3.2.2) Whenever $\mathcal{F} \subseteq \mathbb{L}_{X}$ is a filter such that $\mathcal{F} \subseteq \bigcup_{g \in G} g \mathcal{U}$, there is a $g_{0} \in G$ such that $\mathcal{F} \subseteq g_{0} \mathcal{U}$.

Proposition 3.16. Let $X$ be a countable set and $G=\operatorname{Sym}(X)$. Then none of the subgroups in $\mathbb{M}_{X}$ is PIP.

Proof. In this case $\mathbb{L}_{X}$ is the full powerset of $X$. Let $\mathcal{U}$ be a nonprincipal ultrafilter on $X$. Let $\mathcal{F}$ be a nonprincipal ultrafilter which is not in the set

$$
\mathcal{X}=\{g \mathcal{U}: g \in G\}
$$

There clearly must be a nonprincipal ultrafilter $\mathcal{F} \notin X$ since there are $2^{2^{\aleph_{0}}}$ nonprincipal ultrafilters on $X$ (see [3]) but $|X|=2^{\aleph_{0}}$.

Let $A \in \mathcal{F}$. If $A$ is cofinite, then $A \in \bigcap_{g} g \mathcal{U}$. On the other hand, if $A$ is infinite-coinfinite, we can choose an infinite-coinfinite set $B \in \mathcal{U}$ and a bijection $g: X \rightarrow X$ such that $g B=A$. So $A \in g \mathcal{U}$. This implies that $\mathcal{F} \subseteq \cup_{g} g \mathcal{U}$ but $\mathcal{F} \nsubseteq g \mathcal{U}$ for any $g \in G$.

Corollary 3.17. Let $G=\operatorname{Sym}(X)$ for a countable set $X$. Then there is a pure-injective G-set, none of whose indecomposable components is pureinjective. 
Proof. The pure-injective hull $H(X)=X \amalg\left(\amalg_{H \in \mathbb{I M}_{X}} G / H\right)$ has this property by the above proposition. Note that $X$ itself is not pure-injective: take $S$ as in Proposition 3.4 to be the subgroup of finitary permutations (i.e. $S$ consists of those permutations each of which fixes all but finitely many elements of $X)$; clearly $\operatorname{fix}_{X}(S)=\emptyset$.

The referee has asked whether there is an example with the property of 3.17 and with $G$ countable; we do not see the answer to this and leave it as an open problem.

\section{Definable subcategories and the Ziegler Spec- trum}

\subsection{Definable subcategories of $G$-Set}

Recall that in the non-additive context we say that the definable subcategories are those full subcategories whose objects are models of a coherent theory. For example, for a fixed $g \in G$, the class

$$
\left\{X \in G \text {-Set : } \operatorname{fix}_{X}(g) \neq \emptyset\right\}
$$

forms a definable subcategory of $G$-Set.

Lemma 4.1. The collection of definable subcategories is closed under finite unions and arbitrary intersections.

Proof. Let $\mathbf{D}_{1}=\operatorname{Mod} T_{1}$ and $\mathbf{D}_{2}=\operatorname{Mod} T_{2}$ be two definable subcategories where $T_{1}$ and $T_{2}$ are sets of coherent sentences. Then $\mathbf{D}_{1} \cup \mathbf{D}_{2}=\operatorname{Mod} T$ for

$$
T=\left\{\varphi_{1} \vee \varphi_{2}: \varphi_{1} \in T_{1}, \varphi_{2} \in T_{2}\right\}
$$

Since coherent sentences are closed under disjunctions, we have that $\mathbf{D}_{1} \cup \mathbf{D}_{2}$ is definable.

Now suppose that $\mathbf{D}_{i}=\operatorname{Mod} T_{i}$ for $i \in I$. Then

$$
\bigcap_{i} \mathbf{D}_{i}=\operatorname{Mod}\left(\bigcup_{i} T_{i}\right)
$$

and so $\bigcap_{i} \mathbf{D}_{i}$ is definable. 
If the class of all $G$-sets were a set, then the definable subcategories would be closed sets for a topology on $G$-Set. In the case of modules, definable subcategories are determined by (nonzero) indecomposable pure-injectives ([13, 4.10], or see, e.g., [8, 5.1.1]) and there is only a set of these up to isomorphism. We therefore have a nice (and in some cases completely classifiable [8, Chapters 5 \& 8]) set of modules with a topology whose closed sets correspond to coherent theories. This is the Ziegler spectrum for modules. It is natural to ask whether the same is true of $G$-sets. The first natural set to consider, in view of what is true about modules, is the set of indecomposable pure-injective $G$-sets.

\subsection{A Ziegler spectrum for $G$-sets}

Proposition 4.2. Let $G$ be a finitely generated free abelian group of rank $\geq 2$. Then there is a definable subcategory $\mathbf{D}$ containing a non-trivial G-set such that $\mathbf{D}$ contains no indecomposable $G$-set other than $\{*\}$

Proof. Let $\left\{g_{1}, \ldots, g_{n}\right\}$ be a set of free generators for $G$. Let $H_{1}=\left\langle g_{1}\right\rangle$ and $H_{2}=\left\langle g_{2}, \ldots, g_{n}\right\rangle$ and put

$$
T=\left\{\exists z_{1}\left(g_{1} z_{1}=z_{1}\right), \exists z_{2}\left(z_{2} \in \operatorname{fix}\left(H_{2}\right)\right)\right\}
$$

and let $\mathbf{D}=\operatorname{Mod} T$. Then

$$
\begin{aligned}
G / Q \models T \quad \text { iff } & \exists h_{1}, h_{2} \in G \text { s.t. } H_{1}^{h_{1}} \leq Q \text { and } H_{2}^{h_{2}} \leq Q \\
\text { iff } & H_{1} \leq Q \text { and } H_{2} \leq Q \text { (since } G \text { is abelian) } \\
\text { iff } & Q=G
\end{aligned}
$$

It is clear from this example that definable subcategories are not determined by the indecomposable pure-injectives they contain. For example, suppose the group $G$ above has rank $\geq 3$. If $H_{1}^{\prime}=\left\langle g_{1}, \ldots, g_{n-1}\right\rangle, H_{2}^{\prime}=\left\langle g_{n}\right\rangle$ and $\mathbf{D}^{\prime}=\operatorname{Mod}\left(\operatorname{fix}\left(H_{1}^{\prime}\right) \neq \emptyset \wedge \operatorname{fix}\left(H_{2}^{\prime}\right) \neq \emptyset\right)$, then $\mathbf{D}^{\prime}$ and $\mathbf{D}$ both contain $\{*\}$ as the only indecomposable pure-injective but $\mathbf{D} \neq \mathbf{D}^{\prime}$ since $G / H_{1} \amalg G / H_{2} \in \mathbf{D} \backslash \mathbf{D}^{\prime}$.

So we have seen that the set of indecomposable pure-injectives (or even the set of indecomposables) is too small to function as the underlying set for a "Ziegler topology". Let $\left\{H_{i}: i \in I\right\}$ be the set of all subgroups of a group 
$G$ and consider the following set:

$$
X=\left\{\coprod_{i \in I^{\prime}} G / H_{i}: I^{\prime} \subseteq I \text { s.t. } H_{i} \text { not conjugate to } H_{j} \text { for } i \neq j, i, j \in I^{\prime}\right\}
$$

So $X$ is the set of $G$-sets such that no two indecomposable components are isomorphic.

Lemma 4.3. Definable subcategories are determined by their intersection with the set $\mathcal{X}$.

Proof. Let $X$ be any $G$-set. Let $X^{\prime}$ be the $G$-set whose decomposition consists of exactly one isomorphic copy for each indecomposable $G$-set occuring in the decomposition of $X$. For any subgroup $S \leq G$, $\operatorname{fix}_{X}(S) \neq \emptyset \Leftrightarrow \operatorname{fix}_{X^{\prime}}(S) \neq$ $\emptyset$. It is now easy to see that $X$ and $X^{\prime}$ satisfy exactly the same coherent sentences. So $X \in \mathbf{D}$ if and only if $X^{\prime} \in \mathbf{D}$.

Note that with $X$ and $X^{\prime}$ as in the above proof, we have that $X^{\prime}$ is pure-injective if $X$ is. Let $\operatorname{pinj}(X)$ be the set of pure-injectives in $X$.

Proposition 4.4. Definable subcategories are determined by their intersection with $\operatorname{pinj}(X)$.

Proof. The crucial fact is that if $X \in X$, then also $H(X) \in X$ which can be seen by considering Banaschewski's construction of $H(X)$ described earlier.

Let $\mathbf{D}$ and $\mathbf{D}^{\prime}$ be two definable subcategories such that $\mathbf{D} \cap \operatorname{pinj}(\mathcal{X})=$ $\mathbf{D}^{\prime} \cap \operatorname{pinj}(\mathcal{X})$ and suppose that $\mathbf{D} \neq \mathbf{D}^{\prime}$. By the previous lemma this means that $\mathbf{D} \cap \mathcal{X} \neq \mathbf{D}^{\prime} \cap \mathcal{X}$. Let $X \in \mathbf{D} \cap \mathcal{X}$ such that $X \notin \mathbf{D}^{\prime} \cap \mathcal{X}$. Then $H(X) \in$ $\mathbf{D} \cap \operatorname{pinj}(X)$. Since $X$ is elementary equivalent to $H(X)$ (by Lemma 3.6) and $X \notin \mathbf{D}^{\prime}$, we must have $H(X) \notin \mathbf{D}^{\prime}$ which is a contradiction.

In light of the above proposition, we can define for a group $G$, a topological space $\mathrm{Zg}_{G}$ (the Ziegler spectrum of $G$ ) which has underlying set $\operatorname{pinj}(\mathcal{X}) \backslash\{*\}$ and such that the closed sets are of the form

$$
C(T)=(\operatorname{pinj}(X) \backslash\{*\}) \cap \operatorname{Mod} T
$$

where $T$ ranges over the coherent theories of $G$-sets.

Proposition 4.5. The Ziegler topology for G-sets has a basis of compact open sets. 
Proof. Consider the closed set $C(T)$ where $T=\left\{\varphi_{\lambda}\right\}_{\lambda}$ for coherent sentences $\varphi_{\lambda}$. So

$$
C(T)=\bigcap_{\lambda} C\left(\varphi_{\lambda}\right)
$$

In other words, the open sets of the form $O(\varphi)=\mathrm{Zg}_{G} \backslash C(\varphi)$ give a basis of open sets for $\mathrm{Zg}_{G}$. We claim that each $O(\varphi)$ is compact.

Suppose that $O(\varphi) \subseteq \bigcup_{\lambda} O\left(\varphi_{\lambda}\right)$. So

$$
\operatorname{Mod}(\neg \varphi) \cap \operatorname{pinj}(X) \subseteq \bigcup_{\lambda} \operatorname{Mod}\left(\neg \varphi_{\lambda}\right) \cap \operatorname{pinj}(X)
$$

But this implies (by Proposition 4.4)

$$
\operatorname{Mod}(\neg \varphi) \subseteq \bigcup_{\lambda} \operatorname{Mod}\left(\neg \varphi_{\lambda}\right)
$$

Or, equivalently

$$
\bigcap_{\lambda} \operatorname{Mod}\left(\varphi_{\lambda}\right) \subseteq \operatorname{Mod}(\varphi)
$$

That is,

$$
\left\{\varphi_{\lambda}\right\}_{\lambda} \vdash \varphi
$$

By the compactness theorem for first-order logic, there are $\lambda_{1}, \ldots, \lambda_{n}$ such that

$$
\varphi_{\lambda_{1}}, \ldots, \varphi_{\lambda_{n}} \vdash \varphi
$$

which implies that

$$
O(\varphi) \subseteq \bigcup_{i=1}^{n} O\left(\varphi_{\lambda_{i}}\right)
$$

by retracing the above steps.

Proposition 4.6. Let $G$ be a finite group. Then the points of $\mathrm{Zg}_{G}$ are the $G$-sets in $X \backslash\{*\}$ and each point is locally closed.

Proof. The first statement is clear since for a finite group $G$ every $G$-set is pure-injective. For the second statement, let $X \in \mathcal{X}$ be the $G$-set with indecomposable decomposition

$$
G / H_{1} \coprod \cdots \coprod G / H_{n}
$$


For any subgroup $H \leq G$, the sentence $\exists z \operatorname{Stab}(z)=H$ is definable by the negation of a coherent sentence, namely

$$
\exists z\left(z \in \operatorname{fix}(H) \wedge \neg \bigvee_{h \notin H} h z=z\right)
$$

Let $O(H)$ be the basic open set defined by this sentence. Then we can write

$$
\{X\}=\left(\bigcap_{i=1}^{n} O\left(H_{i}\right)\right) \cap \bigcap\left\{O(H)^{c}: H \text { not conjugate to any } H_{i}\right\}
$$

So the point $X$ is locally closed.

It would be nice to describe explicitly the Ziegler topology for some specific groups. However it seems that it is a hard problem to classify even the PIP subgroups in the simplest nontrivial examples. Consider for example the locally finite group $\mathrm{FSym}(\mathbb{N})$ of finitary permutations on $\mathbb{N}$ (the permutations with finite support). This group has a very simple normal subgroup structure, with a unique normal subgroup (which has index 2), namely, the group of even permutations $A_{\infty}$. It is possible that there are no PIP subgroups other than the finite subgroups and $A_{\infty}$. However, we have not been able to decide this question (even after discussions with group-theorists).

\section{References}

[1] Adámek, J. and Rosický, J., Locally Presentable and Accessible Categories, London Math. Soc. Lecture Notes Ser., Vol. 189, Cambridge University Press, 1994.

[2] Banaschewski, B., Equational Compactness of G-Sets, Canadian Mathematical Bulletin, Vol.17(1), 1974, 11-18.

[3] Keisler, H. J. and Chang, C. C., Model Theory, third edition, Studies in Logic and Foundations of Mathematics Vol. 73, Elsevier Science Publishers B.V., 1990.

[4] Hodges, W., Model Theory, Encyclopedia of Mathematics and Applications, Vol. 42, Cambridge University Press, 1993. 
[5] Jensen, C. U. and Lenzing, H., Model Theoretic Algebra; with particular emphasis on Fields, Rings and Modules, Gordon and Breach, 1989.

[6] Mac Lane, S. and Moerdijk, I., Sheaves in Geometry and Logic, SpringerVerlag, 1992.

[7] Mycielski, J. and Ryll-Nardzewski, C., Equationally compact algebras II, Fund. Math., 61 (1968), 271-281.

[8] Prest, M., Purity, Spectra and Localisation, Cambridge University Press, in press.

[9] Prest, M., Model Theory and Modules, London Math. Soc. Lecture Notes Ser., Vol. 130, Cambridge University Press, 1988.

[10] Rajani, R. and Prest, M., "Model-theoretic imaginaries and coherent sheaves", submitted.

[11] Taylor, W., review of [2], Mathematical Reviews, MR0351954 (50 no.4442).

[12] Wenzel, G. H., "Equational compactness", in Grätzer, G. (ed.), Universal Algebra, Second Edition, Springer-Verlag, 1979, 417-447.

[13] M. Ziegler, Model theory and modules, Ann. Pure Applied Logic, 26 (1984), 149-213. 